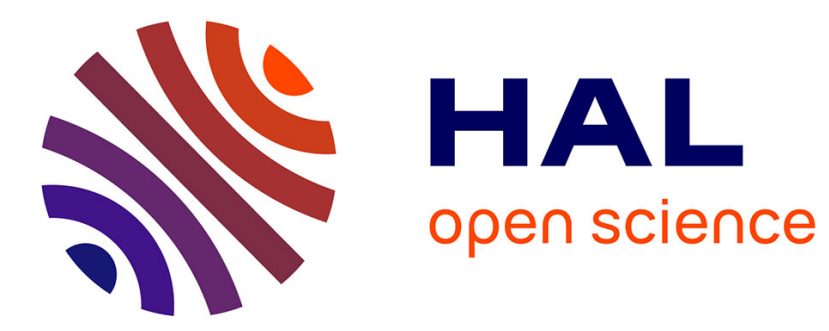

\title{
On spin-wave spectrum of systems without long-range magnetic order
}

\author{
J. Spalek, N. Rivier
}

\section{To cite this version:}

J. Spalek, N. Rivier. On spin-wave spectrum of systems without long-range magnetic order. Journal de Physique Lettres, 1979, 40 (3), pp.57-61. 10.1051/jphyslet:0197900400305700 . jpa-00231569

\section{HAL Id: jpa-00231569 https://hal.science/jpa-00231569}

Submitted on 1 Jan 1979

HAL is a multi-disciplinary open access archive for the deposit and dissemination of scientific research documents, whether they are published or not. The documents may come from teaching and research institutions in France or abroad, or from public or private research centers.
L'archive ouverte pluridisciplinaire HAL, est destinée au dépôt et à la diffusion de documents scientifiques de niveau recherche, publiés ou non, émanant des établissements d'enseignement et de recherche français ou étrangers, des laboratoires publics ou privés. 


\title{
On spin-wave spectrum of systems without long-range magnetic order $\left({ }^{*}\right)$
}

\author{
J. Spałek (**) and N. Rivier \\ Physics Department, Imperial College, London SW7 2BZ, U.K. \\ (Reçu le 23 août 1978, révisé le 18 décembre 1978, accepté le 18 décembre 1978)
}

\begin{abstract}
Résumé. - Nous calculons le spectre d'énergie et l'atténuation des ondes de spin de grandes longueurs d'onde dans des systèmes magnétiques à l'état paramagnétique et dans des verres de spins, en partant des équations de mouvement d'un champ de spin classique $\mathbf{S}(\mathbf{r}, t)$.
\end{abstract}

\begin{abstract}
We summarize briefly our results concerning the calculations of the long-wavelength spin-wave spectrum of magnetic systems in the paramagnetic regime, and of spin glasses, within the classical equation of motion for the spin field $\mathbf{S}(\mathbf{r}, t)$.
\end{abstract}

1. Introduction. - The purpose of this paper is to study the spin-wave spectrum $\omega(\mathbf{k})$ in two systems without magnetic long-range order : a Heisenberg magnet in the paramagnetic regime and a spin glass. In the former case the well developed short-range order (SRO) evolves in time while in the latter we have a random but frozen static configuration specified by angles $\Theta_{0}(\mathbf{r})$ and $\Phi_{0}(\mathbf{r})$ of the local magnetization $\mathbf{S}_{0}(\mathbf{r})$. We start from the classical equation of motion in the long-wavelength limit

$$
\dot{\mathbf{S}}(\mathbf{r}, t)=\mathbf{S}(\mathbf{r}, t) \times\left[\mathbf{B}(\mathbf{r}) \cdot \nabla+C(\mathbf{r}) \nabla^{2}\right] \mathbf{S}(\mathbf{r}, t)
$$

with $B$ and $C$ defined as the first and second moment of the exchange integral $J_{i j}$ (the Hamiltonian is $\left.\mathscr{H}=-\sum_{i j}^{\prime} J_{i j} \mathbf{S}_{i} \cdot \mathbf{S}_{j}\right)$

$$
\begin{aligned}
& \mathbf{B}\left(\mathbf{r}=\mathbf{r}_{i}\right)=\frac{2}{\hbar} \sum_{j(i)} J_{i j} \mathbf{R}_{j i}=\left.\nabla C(\mathbf{r})\right|_{\mathbf{r}=\mathbf{r}_{i}} \\
& C\left(\mathbf{r}=\mathbf{r}_{i}\right)=\frac{1}{\hbar} \sum_{j(i)} J_{i j} \mathbf{R}_{j i}^{2} .
\end{aligned}
$$

Next, we perform the transformation in spin space

$$
\mathbf{S}(\mathbf{r}, t)=A(\mathbf{r}, t) \mathbf{e}_{0}
$$

(*) Post-deadline paper, submitted to the Int. Conf. Low Temperature Physics, LT XV, Grenoble - 1978.

${ }^{* *}$ ) On leave of absence from AGH, Cracow, Poland. to the system of reference in which $\mathbf{S}$ is constant and equal to $\mathbf{e}_{0}$ (we assume $\mathbf{e}_{0}^{2}=1$ ). Such a transformation was constructed for translationally invariant systems ( $B \equiv 0, C$ uniform) by Korenman et al. [1]

$A=\exp \left(-i L_{Z} \Phi\right) \exp \left(-i L_{Y} \Theta\right) \exp \left(-i L_{Z} b\right)$

with $(\Theta, \Phi, b)$ being the Euler angles of rotation from the laboratory system to the system in which $z$ axis is parallel to $\mathbf{S}$, and the generators of the transformation fulfil the angular momentum commutation rules and

$$
L_{i} \mathbf{e}_{j}=i \mathbf{e}_{i} \times \mathbf{e}_{j} .
$$

The resulting equation of motion for the angles $\Theta$ and $\Phi$ are from (1)

$$
\dot{\Theta}=-C(\nabla+\cos \Theta \nabla \Phi+\mathbf{B} / C) \sin \Theta \cdot \nabla \Phi
$$

$$
\sin \Theta . \dot{\Phi}=C(\nabla+\cos \Theta \cdot \nabla \Phi+\mathbf{B} / C) \cdot \nabla \Theta .
$$

One can see that these equations do not depend on the third Euler angle $b$ and therefore one mode $\omega=0$ exists in the system. The eqs. $(7 a-b)$ in the linearized case coincide with those considered by Villain [2].

2. Crystalline case : linearization and solutions. We consider first the case $\mathbf{B} \cong 0$ and $C$ uniform in space. Next, we linearize the nonlinear terms $\sim(\nabla \Phi)^{2}$ and $\sim \nabla \Theta \cdot \nabla \Phi$. The simplest approximation is to consider the presence of a mean field from all excitations acting on one particular mode. Then 
$\cos \Theta \nabla \Theta \cdot \nabla \Phi \simeq \cos \Theta \nabla \Phi \cdot\langle\nabla \Theta\rangle+\operatorname{cotg} \Theta \nabla \Theta \cdot\langle\nabla \Phi \sin \Theta\rangle-\operatorname{cotg} \Theta\langle\nabla \Theta\rangle \cdot\langle\nabla \Phi \sin \Theta\rangle$

$\cos \Theta \sin \Theta(\nabla \Phi)^{2} \simeq 2\langle\operatorname{cotg} \Theta\rangle\langle\nabla \Phi \sin \Theta\rangle(\nabla \Phi \sin \Theta-\langle\nabla \Phi \sin \Theta\rangle)+\operatorname{cotg} \Theta\left\langle(\nabla \Phi \sin \Theta)^{2}\right\rangle$.

We have averaged over quantities $(\nabla \Theta)$ and $(\nabla \Phi \sin \Theta)$ rather than over $\nabla \Theta$ and $\nabla \Phi$ only, since we think they resemble more the spin wave components on a local scale. Assuming that there is no LRO present in the system we will obtain the equation of motion for $\Theta$ when decoupled from $\Phi$

$$
\ddot{\Theta}+C^{2} \nabla^{4} \Theta+2 \alpha \dot{\Theta}-R(\mathbf{r}, t)=0
$$

where the viscous damping constant

$$
\alpha=\frac{1}{2}|C|\left\langle(\nabla \Theta)^{2}\right\rangle,
$$

and $R(\mathbf{r}, t)$ is the random force arising from the nonlinear terms neglected in this approximation. Thus we arrive at the Langevin equation for random motion [3] with restoring force $\sim C^{2} \nabla^{4} \Theta$. This is in agreement with the intuitive expectation that the motion of the spin in the paramagnetic region is a random process modulated by the mutual interactions with its neighbours. The homogeneous part of eq. (9) has a plane wave solution

$$
\Theta \sim \exp [i(\omega t-\mathbf{k . r})]
$$

and gives the dispersion relation in the ferromagnetic case (i.e. for $J<0$ )

$$
\omega^{2}=C^{2} k^{4}+2 i \alpha \omega .
$$

Since in the translationally invariant systems $k$ is real we have $\omega=\omega_{1}+i \omega_{2}$ and therefore two solutions are possible :

$$
\text { 1) } \begin{aligned}
\omega_{2} & =\alpha \\
\omega_{1 \mathbf{k}} & =\left(C^{2} k^{4}-\alpha^{2}\right)^{1 / 2} \\
\text { 2) } & \omega_{1}=0 \\
\omega_{2 \mathbf{k}} & \equiv \omega_{2-}=\alpha-\left(\alpha^{2}-C^{2} k^{4}\right)^{1 / 2} .
\end{aligned}
$$

The solution 1) exists for $k \geqslant k_{\mathrm{c}}$ while 2) for $k \leqslant k_{\mathrm{c}}$ where

$$
k_{\mathrm{c}}=(\alpha /|C|)^{1 / 2} .
$$

So there are propagating modes for $k>k_{\mathrm{c}}$ and only diffusive modes for $k \leqslant k_{\mathrm{c}}$. Additionally, since

$$
\alpha \sim\left\langle(\nabla \Theta)^{2}\right\rangle \sim k_{\mathrm{B}} T
$$

we have $k_{\mathrm{c}} \sim T^{1 / 2}$. Equation (1), when generalized to a difference form to include short wavelength excitations, yields the dispersion relation for a ferromagnet [4]

1)

$$
\omega_{1 \mathbf{k}}=\left\{[J(0)-J(\mathbf{k})]^{2}-\alpha^{2}\right\}^{1 / 2} ; \quad \omega_{2}=\alpha,
$$

2)

$$
\omega_{1}=0 ; \quad \omega_{2-}=\alpha-\left\{\alpha^{2}-[J(0)-J(\mathbf{k})]^{2}\right\}^{1 / 2},
$$

and for an antiferromagnet

1)

$$
\omega_{1 \mathbf{k}}=\left[J^{2}(0)-J^{2}(\mathbf{k})-\alpha^{2}\right]^{1 / 2} ; \quad \omega_{2}=\alpha,
$$

2)

$$
\omega_{1}=0 ; \quad \omega_{2-}=\alpha-\left[\alpha^{2}-J^{2}(0)+J^{2}(\mathbf{k})\right]^{1 / 2} .
$$

In the second case $k_{\mathrm{c}} \sim T$. We refer the reader to the paper [4] for a detailed discussion of these results. Let us mention only that the results are in agreement with the experimental data for $1 \mathrm{~d}$ systems [5] (i.e. existence of propagating modes above $k_{\mathrm{c}}$ and halfwidth $\sim k^{2}$ as $k \rightarrow 0$ for antiferromagnets).

At $T=0, k_{\mathrm{c}}$ and $\alpha$ both vanish and the standard spin-wave dispersion relation is recovered (see e.g. [5]). Additionally, besides (12) and (14), there exists a second diffusive mode with

$$
\omega_{2 \mathbf{k}}=\omega_{2+}=\alpha+\{\cdots\}^{1 / 2},
$$

but since for $k=0$ it follows from (7) that $\omega_{20}=0$, it is a nonphysical branch of excitations.

3. Spin glasses. - Suppose that

$$
\begin{gathered}
\Theta(\mathbf{r}, t)=\Theta_{0}(\mathbf{r})+\delta \Theta(\mathbf{r}, t) \\
\Phi(\mathbf{r}, t)=\Phi_{0}(\mathbf{r})+\delta \Phi(\mathbf{r}, t)
\end{gathered}
$$

with $\delta \Theta \ll \Theta_{0}$ and $\delta \Phi \ll \Phi_{0}$.

Then eqs. $(7 a-b)$ can be rewritten in the form

$$
\begin{array}{r}
-\frac{1}{C} \dot{\delta \Theta}=\delta \Theta\left[(\nabla+\mathbf{B} / C) \cdot\left(\cos \Theta_{0} \nabla \Phi_{0}\right)-\sin ^{2} \Theta_{0}\left(\nabla \Phi_{0}\right)^{2}\right]+\left[\sin 2 \Theta_{0} \nabla \Phi_{0}+\cos \Theta_{0} \nabla \Theta_{0}\right] \cdot \nabla(\delta \Phi)+ \\
+\sin \Theta_{0}(\nabla+\mathbf{B} / C) \cdot \nabla(\delta \Phi)+\cos \Theta_{0} \nabla \Phi_{0} \cdot(\nabla+\mathbf{B} / C) \delta \Theta \\
\frac{1}{C} \dot{\delta} \Phi \sin \Theta_{0}=\left[\cos \Theta_{0} \nabla(\delta \Phi)-\delta \Theta \sin \Theta_{0} \cdot \nabla \Phi_{0}\right] \cdot \nabla \Theta_{0}+\left(\nabla+\cos \Theta_{0} \nabla \Phi_{0}+\mathbf{B} / C\right) \cdot \nabla(\delta \Theta)
\end{array}
$$


We want to solve eqs. (16)-(17) in the long-wavelength limit $k \xi \ll 1$ with $\xi$ being the coherent length of angular distribution of the static magnetization $\mathbf{S}_{0}(\mathbf{r})$. In this limit the propagation for a given degree of freedom (e.g. $\Theta$ ) can be calculated by decoupling it from other degree of freedom $(\Phi)$ and next averaging over static disorder. In such a case we get from (1)

$$
\ddot{\delta} \dot{\mathbf{S}}=-\overline{\mathfrak{L}^{2}} \boldsymbol{\delta} \mathbf{S}
$$

or equivalently

$$
\begin{aligned}
\dot{\delta} \dot{\Theta}+\overline{C^{2}} \nabla^{4} \delta \Theta+\left\langle\left[\frac{5}{3} C \nabla^{2} C\right.\right. & \left.\left.+\frac{4}{3}(\nabla C)^{2}\right]\right\rangle_{\mathrm{c}} \times \\
& \times \nabla^{2} \delta \Theta=0
\end{aligned}
$$

where $\langle\cdots\rangle_{\mathrm{c}}$ means an average over all possible configurations of disorder and

$$
\overline{C^{2}}=\left\langle C^{2}\right\rangle_{\mathrm{c}}, \quad \overline{\mathfrak{L}^{2}}=\left\langle\left(C \nabla^{2}+\mathbf{B} \cdot \nabla\right)^{2}\right\rangle_{\mathrm{c}} .
$$

\section{Defining}

$$
\overline{C^{2}} q_{0}^{2} \equiv-\left\langle\frac{5}{3} C \nabla^{2} C+\frac{4}{3}(\nabla C)^{2}\right\rangle
$$

and substituting a plane wave solution for $\delta \Theta$ one obtains

$$
\omega=\sqrt{\overline{C^{2}}} k\left(q_{0}^{2}+k^{2}\right)^{1 / 2} .
$$

If $q_{0}^{2}<0$ then in the limit $\mathbf{k} \rightarrow 0$ only diffusive modes exist (cf. [6]). Propagating modes will be normal modes of the system for $k>\left|q_{0}\right| \sim q_{\mathrm{c}}=2 \pi / \xi$. But from (19) one can see that this is unlikely since in order to calculate the average in (19) we assume again that the details of disordered structure for $k \xi \ll 1$ are unimportant and therefore one can admit that

$$
C \sim \sin \mathbf{q}_{\mathrm{c}} \cdot \mathbf{r} .
$$

This yields the linear dispersion relation for $k \rightarrow 0$, $\omega=A|\mathbf{k}|$ with positive exchange constant

$$
A=\left(\overline{C^{2}}\right)^{1 / 2} q_{0}=\left(\overline{C^{2}}\right)^{1 / 2} q_{\mathrm{c}} / \sqrt{3} .
$$

We would like to underline that, as previous approaches $[10,11]$ our result $(20)$ is proved in the situation in which only a smooth space variation of $\mathbf{S}_{0}(\mathbf{r})$ is present [12]. For a particular case of ferromagnet we recover $\omega=\sqrt{\overline{C^{2}}} k^{2}$. Additionally, since $D$ is determined by disorder there is no simple relation of our linear in | $\mathbf{k}$ | spin-wave spectrum with the corresponding one of an antiferromagnet or of the Mattis model of spin glass [7].

Let us include the damping in (18) in a phenomenological way. Spin glasses exhibit magnetic viscosity [8] so in the simplest case the damping is similar to that usually postulated for highly viscous liquids and glasses $[9 a]$ and therefore (18) becomes

$$
\ddot{\delta \Theta}+\overline{C^{2}} \nabla^{2}\left(\nabla^{2}-q_{0}^{2}\right) \delta \Theta+2 \alpha \dot{\delta \Theta}=0 .
$$

The dispersion relation is then

$$
\omega^{2}=\overline{C^{2}} k^{2}\left(q_{0}^{2}+k^{2}\right)+2 i \alpha \omega
$$

which for $\omega=\omega_{1}+i \omega_{2}$ has two solutions as in the previous section

1)

$\omega_{1 \mathbf{k}}=\left[\overline{C^{2}} k^{2}\left(k^{2}+q_{0}^{2}\right)-\alpha^{2}\right]^{1 / 2} ; \quad \omega_{2}=\alpha$

2)

$\omega_{1}=0 ; \quad \omega_{2-}=\alpha-\left[\alpha^{2}-\overline{C^{2}} k^{2}\left(q_{0}^{2}+k^{2}\right)\right]^{1 / 2}$.

The first solution exists for $k \geqslant k_{\text {c }}$ while the second for $k \leqslant k_{\mathrm{c}}$ with $k_{\mathrm{c}}$ given by

$$
k_{\mathrm{c}}=-\frac{1}{2} q_{0}^{2}+\left[\frac{1}{4} q_{0}^{2}+\alpha^{2} / \overline{C^{2}}\right]^{1 / 2} .
$$

For small $\omega$ the term $\sim \omega^{2}$ in (24) can be neglected and we get $\omega \sim i k^{2}$ in agreement with the result of Dzialoshinskii and Volovik [10].

Let us now discuss a damping in a more systematic way and compare our results with those of a hydrodynamical approach, $\omega=A|\mathbf{k}|+i D \mathbf{k}^{2}$ [11]. We must emphasize that the choice of a phenomenological damping term depends on the particular situation of the system under consideration. Halperin and Saslow [11] assumed, as in ordinary liquids $[9 b]$ that the last term in (23) should be represented by

$$
\text { - } D \nabla^{2}(\dot{\delta} \dot{\Theta}),
$$

so effectively in (24) $\alpha=\frac{1}{2} D k^{2}$. This form of damping is based on the assumption that the mode with $k=0$ corresponding to the uniform motion or rotation (in case of spin system) of the system as a whole is not damped. If, however, the mode with $k=0$ is damped (through the coupling of the spin system to other degrees of freedom, electrons, etc.), and if the relaxation takes place to one (or a few) well defined equilibrium (frozen) configurations, then, in full generality, the expansion of the relaxation term is of the form

$$
\begin{aligned}
& \tilde{R}\{\delta \Theta\}=\alpha_{0}^{2} \delta \Theta+2 \alpha_{1} \dot{\delta \Theta}-\beta_{1} \nabla^{2}(\dot{\delta \Theta})+\cdots+ \\
& \quad+\beta_{n}\left(\nabla^{2}\right)^{n} \dot{\delta \Theta}+\cdots+\text { nonlinear terms } \equiv \alpha_{0}^{2} \delta \Theta \\
& +2 \alpha \dot{\delta}+\text { nonlinear terms }
\end{aligned}
$$

where $\alpha=\alpha_{1}+\frac{1}{2} \beta_{1} k^{2}+\cdots$, and with $\tilde{R}\{\delta \Theta\}$ replacing the last term in (23). In (28) the terms proportional to the parameters $\alpha_{0}$ and $\alpha_{1}$ reflect the relaxation to well defined equilibrium position and the coupling of the spin subsystem (moving uniformly) to the other degrees of freedom like conduction electrons and/or the lattice. In particular, one can easily show that for the relaxation in the Bloch-Bloembergen form $\alpha_{0}=\alpha_{1}$ and $\beta_{1}=\beta_{2}=\cdots=0$ [4]. On the other hand, the terms containing $\beta^{\prime}$ s start from the one $\sim \beta_{1} \nabla^{2}(\dot{\Theta} \Theta)$ which expresses usual lowest order hydrodynamical damping due to the relative motion 
of neighbouring parts within the spin subsystem only. The expansion is odd in time and even in space derivatives and since second order time derivatives determine the equation of motion completely only first order time derivatives will enter in (28). The presence of even order space derivatives only is caused by the fact that we assume that there is the inversion symmetry $\mathbf{x} \rightarrow-\mathbf{x}$ of the relative motion of two parts of the system. If we take into account the relaxation processes within the spin subsystem only, and if it takes place to the rotationally invariant ground state then $\alpha_{0}=\alpha_{1}=0$, the damping to lowest order in $k$ is of a hydrodynamic type [11]. Assuming, however, that (28) is the most general form of relaxation term appropriate for a spin glass, we find the solutions

1) $\left(k \geqslant k_{\mathrm{c}}\right)$

$\omega_{1 \mathbf{k}}=\left[\overline{C^{2}} \mathbf{k}^{2}\left(q_{0}^{2}+\mathbf{k}^{2}\right)-\alpha^{2}+\alpha_{0}^{2}\right]^{1 / 2} ; \quad \omega_{2}=\alpha$.

2) $\left(k \leqslant k_{\mathrm{c}}\right)$

$\omega_{1}=0$;

$\omega_{2 \mathbf{k}}=\omega_{2-}=\alpha-\left[\alpha^{2}-\alpha_{0}^{2}-\overline{C^{2}} \mathbf{k}^{2}\left(q_{0}^{2}+\mathbf{k}^{2}\right)\right]^{1 / 2}$

with $k_{\mathrm{c}}$ given by the condition $\omega_{1 \mathbf{k}_{\mathrm{c}}}=0$. For the hydrodynamic damping (i.e. $\alpha=1 / 2 \beta_{1} k^{2}$, and $\alpha_{0}=0$ ) we obtain from (29) the Halperin and Saslow [11] dispersion relation $\omega_{1 \mathbf{k}}=A|\mathbf{k}| ; \omega_{2}=D \mathbf{k}^{2}$, with $A=\left(\overline{C^{2}}\right)^{1 / 2}\left|q_{0}\right|$ and $D=\frac{1}{2} \beta_{1}$ in the lowest order in $k$, whereas $k_{\mathrm{c}}=0$.

4. Interpretation of the results. - We have applied the classical equation of motion (1) and its generalization to short wavelength limit to study the spinwave excitations in the two systems without long-range magnetic order : a Heisenberg paramagnet and a spin glass. The initial eqs. (7a-b) have first been linearized which means that we include only the influence of the average amplitude of all excitations present in the thermal background of the system onto one particular mode. This is an approximation equivalent to the RPA in the ordered region and we expect it to be valid for not strongly fluctuating Heisenberg magnet in a disordered region, e.g. one- or two-dimensional magnets at low temperature. The resulting viscous damping introduced in this way reflects a random rotation of a given spin modulated by the interaction with its nearest neighbours. The modes are diffusive for $k \leqslant k_{\mathrm{c}}$ and propagating above that value. This can be interpreted as follows : if we define $\omega_{\mathbf{k}}(0)$ as $\omega_{1 \mathrm{k}}$ for $T=0(\alpha=0)$ then the spin waves with energy $\omega_{\mathbf{k}}(0) \leqslant k_{\mathrm{B}} T$ are not distinguishable from the thermal noise present in the statistical equilibrium at non zero temperature. Additionally, the two diffusive modes for $k \rightarrow 0$ behave like $\omega_{2+} \sim 2 \alpha$ and $\omega_{2-} \sim 0\left(k^{4}\right)$ for ferromagnet. Since for the pure Heisenberg system the uniform mode $(k=0)$ is not damped

$$
(\Theta=\Phi=\text { const. })
$$

we have that only mode $\omega_{2-}$ is excited. Therefore, our results are in disagreement with those of Halperin and Hohenberg [13] who predicted only diffusive modes in the paramagnetic region with $\omega_{2} \sim k^{2}$. The results obtained from the hydrodynamical approach [13] however are in clear contradiction with what is observed experimentally for quasi-one dimensional systems [5] and for 3d magnets [15]. Our results in the short-wavelength limit reduce to those obtained recently from the low temperature expansion by Reiter and Sjölander [14] (see [4] for a full discussion).

Summarizing, our approach may be viewed as a simple analytic approach via the classical equation of motion of the Landau-Lifshitz type with inclusion of the damping appropriate to the situation with rotationally invariant ground state which is in qualitative agreement both with the experimental data [5] and with the exact perturbation expansion [14].

In the second part (sect. 3) we have solved the eqs. $(7 a-b)$ in the case of long-wavelength excitations in a spin glass which is defined by frozen but random static configuration of spins. In this case the decomposition $\mathbf{S}(\mathbf{r}, t)=\mathbf{e}_{0}(\mathbf{r})+\mathbf{\delta} \mathbf{S}(\mathbf{r}, t)$ such that

$$
\|\mathbf{S S}(\mathbf{r}, t)\| \ll 1
$$

is possible and an explicit form of equation of motion for the dynamic part $\boldsymbol{\delta S}(\mathbf{r}, t)$ is given by (16) and (17). To discuss the solutions for long wavelengths we average these equations over all static configuration of disorder. This introduces an effective medium and reflects the fact that the long-wavelength excitations see many short-range order static configurations over one wavelength (i.e. $k \xi \ll 1$ ). Secondly, since the simple linearization $(8 a-b)$ is not applicable in this case (we have linearized the system already by the decomposition into static $e_{0}(\mathbf{r})$ and dynamic parts $\delta \mathbf{S}(\mathbf{r}, t)$ ) we must introduce the damping phenomenologically as it is discussed in sect. 3 . The results show that the effective exchange $D$ is positive and if we neglect in the damping term the coupling to other degrees of freedom (conduction electrons, the lattice), then the results reduce to those of Halperin and Saslow [11] in the first nontrivial order in $\mathbf{k}$. Furthermore, on the basis of our general results (29) and (30) one can see that the long-wavelength excitations are observable if the homogeneous part of viscous damping ( $\left.\sim 2 \alpha_{1} \dot{\delta \Theta}\right)$ is sufficiently small, i.e. there exist regions of $k>k_{\mathrm{c}}$ such that $k \xi \ll 1$ and simultaneously $\omega_{1 \mathbf{k}}>\omega_{2}$.

Acknowledgments. - We are grateful to the referees for very useful critical remarks which led to the substantial improvement of our paper. 


\section{References}

[1] Korenman, V., Murray, J. L. and Prange, R. E., Phys. Rev. B 16 (1977) 4058.

[2] Villain, J., J. Phys. C 6 (1973) L97.

[3] Kubo, R., Rep. Prog. Phys. 29 (1966) 255.

[4] SpazeK, J., to be submitted.

[5] Steiner, M., Villain, J. and Windsor, C. G., Adv. Phys. 25 (1976) 87.

[6] Rivier, N., Wiss. Z. Tech. Univers. Dresden 23 (1974) 1000.

[7] Sherrington, D., J. Phys. C 10 (1977) L7.

[8] Guy, C. N., J. Phys. F 8 (1978) 1309.

[9] LANDAU, L. and LifSHITZ, E. M., (a) Theory of Elasticity, $\S 31,29 ;$ (b) Fluid Mechanics, § 15 (Pergamon Press, London) 1959 .
[10] Dzialoshinski,, J. E. and Volovik, G. E., J. Physique 39 (1978) 693.

[11] Halperin, B. I. and Saslow, W. M., Phys. Rev. B 16 (1977) 2154.

[12] SPAzEK, J. and Rivier, N., to be submitted.

[13] Halperin, B. I. and Hohenberg, P. C., Phys. Rev. 188 (1969) 898.

[14] Reiter, G. and Sjolander, A., Phys. Rev. Lett. 39 (1977) 1047

[15] Lynn, J. W., Phys. Rev. B 11 (1975) 2624;

Evans, M. T. and Windsor, C. G., J. Phys. C 6 (1973) 495. 